\title{
A Preclinical Investigation into the Effects of Aging on Dermal Hyaluronan Properties and Reconstitution Following Recombinant Human Hyaluronidase PH20 Administration
}

\author{
Robert J. Connor - Barbara Blouw · Jessica Cowell • Kelly Chen • \\ Chunmei Zhao · David W. Kang
}

Received: January 24, 2020 / Published online: May 2, 2020

(C) The Author(s) 2020

\section{ABSTRACT}

Introduction: There is currently no consensus in the literature concerning the impact of aging on the properties of hyaluronan (HA) in the subcutaneous (SC) space. Recombinant human hyaluronidase $\mathrm{PH} 20$ (rHuPH20) facilitates SC administration of injected therapeutics by depolymerizing SC HA, facilitating bulk fluid flow, dispersion and absorption. This study assessed the impact of intrinsic aging on $\mathrm{HA}$ in the SC space and thus the ability of rHuPH20 to enhance delivery of co-administered therapeutics.

Methods: Histologic evaluations of HA levels and degradation were performed on human skin samples from six age groups, aged from 20 to 100 years. HA levels were evaluated by HA staining and degradation by staining samples for HA following incubation with rHuPH20. HA was extracted from samples and HA size determined by gel electrophoresis. Dermal

Digital Features To view digital features for this article go to https://doi.org/10.6084/m9.figshare.12123597.

R. J. Connor · B. Blouw · J. Cowell · K. Chen ·

C. Zhao · D. W. Kang $(\bowtie)$

Halozyme Therapeutics, Inc., San Diego, CA 92121, USA

e-mail: publications@halozyme.com reconstitution was assessed in young (aged 1.5 months) and elderly (aged $>16$ months) mice. Baseline dye dispersion was measured at 5 and $20 \mathrm{~min}$ post-intradermal dye injection. Following treatment with rHuPH20, dye dispersion was measured again at 2, 24, 48, 72 and $96 \mathrm{~h}$.

Results: Distribution of HA was confined to the interstitial space between adipocytes, with similar pericellular presence and levels of HA found across all age groups. Substantial levels of highmolecular-weight HA were observed in all age groups at baseline. Incubation with a clinically relevant dose of rHuPH20 resulted in degradation of all SC HA and similar degradation profiles independent of age. No difference in dye dispersion time was observed between young and elderly mice across the range of time points assessed, with dye dispersion returning to baseline levels by $24 \mathrm{~h}$ after rHuPH20 treatment.

Conclusions: Subcutaneous delivery of approved therapeutics facilitated by co-administration with rHuPH20 should not be impacted by intrinsic aging, with this study providing no evidence for an effect of aging on HA distribution, structure or a loss of rHuPH20 efficacy.

Keywords: Aging effect; Dermal reconstitution; Drug delivery; Enzymatic degradation; Hyaluronan distribution; Hyaluronidase; rHuPH20; Subcutaneous injection 


\section{Key Summary Points}

Why carry out this study?

Recombinant human hyaluronidase PH20 (rHuPH20) facilitates subcutaneous administration of large volumes of coadministered therapeutics by depolymerizing hyaluronan (HA) in the subcutaneous space, thus enabling bulk fluid flow and facilitating dispersion and absorption.

Intrinsic aging may affect the levels and distribution of HA in the subcutaneous space, thereby affecting the ability of rHuPH20 to enhance delivery of coadministered therapeutics.

This study assessed the effect of aging on HA distribution, size and degradation in human skin samples and dermal reconstitution of HA after $\mathrm{rHuPH} 20$ treatment in young and elderly mice.

\section{What was learned from the study?}

This study found no evidence for an effect of aging on HA distribution, size, degradation or dermal reconstitution, and there was no apparent loss of rHuPH20 effectiveness with increasing age.

Subcutaneous delivery of approved therapeutics facilitated by coadministration with rHuPH20 should not be impacted by intrinsic aging.

\section{INTRODUCTION}

Administration of therapeutics via intravenous (IV), intramuscular or subcutaneous (SC) injection is one of the most common healthcare procedures worldwide [1, 2]. While IV injection has traditionally been the standard route of administration for many therapeutics, SC delivery offers many potential advantages. Notably, SC administration may be more convenient than IV, owing to shorter administration times [3], potentially reduced infusionrelated reactions [4] and the ability of SC formulations to be self-administered at home $[5,6]$. These factors not only act to reduce the burden on patients and caregivers, the healthcare system and healthcare professionals (HCPs), but often lead to SC delivery being preferred by patients and HCPs alike $[3,7,8]$.

Despite the potential benefits, rapid SC administration is limited by low delivery volumes and flow rates as a result of the physiology of the SC space $[9,10]$. This region, located between the dermis and the underlying muscle layer, consists of adipose tissue bound by an extracellular matrix (ECM) that physiologically limits the volume and rate of fluid that can be injected. Constituents of the ECM include glycosaminoglycans (GAGs), collagen and elastin fibers, and lymphatic and blood vessels. While lymphatic and blood vessels are essential for bodily function, and collagen has a half-life of almost 15 years [11], some GAGs are enzymatically degradable and have a high turnover rate, with the principal GAG of the hypodermis, hyaluronan (HA), being reconstituted $24-42 \mathrm{~h}$ following enzymatic degradation [12]. In the ECM, HA forms a gel-like substance with water, creating a barrier to fluid flow $[13,14]$, and plays a crucial role in limiting large-volume SC drug delivery and restricting drug dispersion and absorption. These properties, combined with its rapid turnover, make $\mathrm{HA}$ a key target for improving the efficiency of SC delivery.

Recombinant human hyaluronidase PH20 (rHuPH20) has proven effective in facilitating large volumes of therapeutics to be delivered rapidly by SC administration by targeting HA [15]. Its use is currently approved in a number of SC formulations of therapeutics [16]. $\mathrm{rHuPH} 20$ is a genetically engineered soluble form of the naturally occurring human hyaluronidase PH20 enzyme, which functions through the enzymatic depolymerization of HA [17]. Its action enables bulk fluid flow in the interstitial space and facilitates the dispersion of injected therapeutics to capillaries and lymphatic vessels $[17,18]$.

Currently, it is not known whether the ability of rHuPH20 to enhance delivery of co- 
administered therapeutics is impacted by changes to the levels, distribution and polymer size of $\mathrm{HA}$ in the SC space. Aging causes a number of known structural changes to human skin [19], with studies showing that dermal HA may also be affected [20-24]. With aging, HA becomes progressively more tissue-bound [21] and has the potential to be deacetylated [20]. To date, there is no consensus in the literature concerning the effect of aging on the overall level of HA in the SC space [21-23] or how the size of SC HA polymers may be impacted by aging [20, 24]. In addition, age-related changes in HAS and $H Y A L$ gene expression may alter or reduce the levels of HA that are present in the SC space [25]. The effects of these changes in levels, distribution and size of SC HA as well as the impact on the ability of the rHuPH20 enzyme to recognize and degrade its substrate have not been characterized. Thus, it would be valuable to determine whether the levels, distribution and size of HA in the SC region change with age and whether potential age-related modifications to $\mathrm{HA}$ alter the ability of rHuPH20 to deliver the co-administered therapeutic.

The aim of this study was to assess the impact of aging on HA levels, distribution and polymer size in human skin samples from donors 20-100 years of age. The study was also designed to elucidate any effect of aging on the ability of rHuPH20 to enhance delivery of coadministered therapeutics by measuring $\mathrm{HA}$ degradation following incubation with rHuPH20 in these human skin samples. Furthermore, HA reconstitution times were assessed following rHuPH20 administration in young and elderly mice.

\section{METHODS}

\section{Histologic Analyses}

Human full-thickness skin samples from donor cadavers were obtained from the National Disease Registry Interchange (NDRI, Philadelphia, PA, USA; protocol no. DZEM1) who are supported by NIH grant U42OD111158. All samples were recovered in full respect of the 1964 Declaration of Helsinki and its later amendments, following approval by the University of Pennsylvania Institutional Review Board (IRB) and according to institutional regulations and guidelines regarding informed consent and donor confidentiality. The skin samples spanned six age intervals: 20-45, $46-55,56-65,66-75,76-85$ and $86-100$ years, with two male and two female samples per age group. The tissues were freshly preserved postmortem, and formalin-fixed paraffin-embedded (FFPE) tissue sections $(5 \mu \mathrm{M})$ were prepared for histologic evaluation of HA levels and degradability in the SC space.

\section{Detection and Analysis of HA from Human Skin Samples}

Formalin-fixed, paraffin-embedded slides were prepared from each tissue sample. For HA staining, sections were deparaffinized, hydrated and stained using a biotinylated TSG6- $\Delta \mathrm{Hep}-\mathrm{Fc}$ probe (HTI-601) followed by hematoxylin counterstaining [26]. Visual evaluation of run controls was performed prior to HA quantification on the human skin samples to confirm successful runs. Run controls consisted of an FFPE section derived from naturally HA-accumulating human xenograft tissue. Representative micrographs were captured using an Aperio ImageScope version 12.2.1.5005 scanner (Leica Biosystems Inc., San Diego, CA, USA). For HA quantification, a digital scoring algorithm was used to quantify the HA content of tissue sections (Aperio Positive Pixel Count V9, Leica Biosystems Inc., San Diego, CA, USA). For each tissue section, a representative region of interest (ROI) within the SC region was analyzed and HA content was calculated as percentage of HApositive pixels over the entire pixel count in the ROI (only stained tissue is included).

\section{Degradability of HA Using rHuPH20}

For assessment of HA degradation, tissue sections $(5 \mu \mathrm{m})$ were treated with $\mathrm{rHuPH} 20$ at $1 \mu \mathrm{g} / \mathrm{ml}$ $(110 \mathrm{U} / \mathrm{ml})$ and $18 \mu \mathrm{g} / \mathrm{ml}(2000 \mathrm{U} / \mathrm{ml})$ for $1 \mathrm{~h}$ at $37^{\circ} \mathrm{C}$ in a humidified chamber using a PIPES buffer ( $25 \mathrm{mM}$ PIPES, $70 \mathrm{mM} \mathrm{NaCl}, 0.5 \%$ bovine serum albumin [BSA], pH 5.5) followed by HA staining and HA quantification as described 
above. To assess differences in degradation, a time-course study was performed using a low enzyme concentration $(0.1 \mu \mathrm{g} / \mathrm{ml} ; 11 \mathrm{U} / \mathrm{ml})$. Slides were incubated for $1,1.5$ or $2 \mathrm{~h}$ before being stained and scored for HA. Enzyme dilutions were performed using PIPES buffer.

\section{Extraction and Size Determination of HA from SC Tissue}

HA was extracted from FFPE human SC tissue by removing approximately $100 \mathrm{mg}$ of tissue from each skin sample and incubating it in proteinase $\mathrm{K}(1 \mathrm{mg} / \mathrm{ml}$, in buffer containing $50 \mathrm{mM}$ Tris- $\mathrm{HCl} \mathrm{pH} 8.0,10 \mathrm{mM} \mathrm{MgCl}_{2}, 2 \mathrm{mM} \mathrm{CaCl}$ ) for $15 \mathrm{~h}$ at $55^{\circ} \mathrm{C}$. Proteinase $\mathrm{K}$ was then inactivated by incubation at $100{ }^{\circ} \mathrm{C}$ for $30 \mathrm{~min}$. The sample was centrifuged at $10,000 \times g$ for $20 \mathrm{~min}$ at $4-10{ }^{\circ} \mathrm{C}$ to pellet tissue debris, and then the supernatant was removed, transferred to a new tube and stored at $-80{ }^{\circ} \mathrm{C}$ until analyzed. To assess the size of the HA polymers extracted from the SC space, $5 \mu \mathrm{g}$ of each sample was separated on a $0.4 \%$ agarose gel $(100 \mathrm{~V} ; 1.25 \mathrm{~h})$. Samples were transferred overnight to a Hybond $^{\mathrm{TM}}-\mathrm{N}+$ membrane (GE Healthcare, Amersham, UK) and blocked using 2\% nonfat dry milk in $0.05 \%$ Tween-20. A biotin-TSG6$\Delta$ Hep-Fc probe [27] $(0.5 \mu \mathrm{g} / \mathrm{ml})$ was used to bind HA molecules on the membrane, and horseradish peroxidase (HRP)-conjugated streptavidin was used for detection.

\section{Dermal Reconstitution Dye Dispersion Assay in Mice}

A total of 52 athymic mice were used for all in vivo studies. All procedures conducted on research animals were approved by the Halozyme Institutional Animal Care and Use Committee (IACUC) and complied with the Halozyme IACUC protocol no. 2017-06C. Comparison of dye dispersion in treated and untreated skin at successive intervals post-enzyme injection provided an estimate of the HA reconstitution time after $\mathrm{rHuPH} 20$ treatment. Of the 52 athymic mice, 26 were obtained and held on-site for $>16$ months for use in the study (elderly mice), and 26 strain-matched
Fig. 1 HA levels in SC tissue from human skin demonstrated maintained levels across all age groups. a Tissue section showing pericellular HA present in all age brackets. HA is shown stained brown using a biotinylated TSG6$\Delta \mathrm{Hep}-\mathrm{Fc}$ probe (HTI-601) followed by hematoxylin counterstain. b Quantification of HA level was performed for all samples by selecting a ROI in the SC space and measuring the \%HA-positive pixels vs. total pixel count. c Western blot detection of SC HA (following Proteinase $\mathrm{K}$ treatment) using a biotinylated TSG6 probe showed a similar distribution pattern of HA size across age groups. $H A$ hyaluronan, $R O I$ region of interest, $S C$ subcutaneous, $S E M$ standard error mean

young mice ( $\sim 1.5$ months of age) were used for comparison of dye dispersion. Each group received a $0.04 \mathrm{ml}$ intradermal injection of $0.4 \%$ Trypan Blue (Corning ${ }^{\circledR}$ catalog no. 25-900-CL) to the right flank of the animals to assess baseline dye dispersion (the amount of dye dispersion that occurs with no rHuPH20 pretreatment). Injection sites were photographed (Nikon, Melville, NY, USA) utilizing a laser distance meter (Leica Geosystems, Norcross, GA, USA) to maintain the same magnification. The area of dye dispersion was then measured at 5 and $20 \mathrm{~min}$ post-injection. Animals were allowed to recover and then received an intradermal injection of rHuPH20 $(0.04 \mathrm{ml} ; 2000$ $\mathrm{U} / \mathrm{ml}$ ) to the left flank. At timepoints 2, 24, 48, 72 and $96 \mathrm{~h}$ following rHuPH20 treatment, animals received a $0.04 \mathrm{ml}$ intradermal injection of $0.4 \%$ Trypan blue dye to the left flank, and dye dispersion was measured at 5 and 20 min post-dye injection. Dermal dye dispersion area was assessed using an Image-Pro Analyzer 7.0 (Media Cybernetics, Inc., Bethesda, MD, USA). Images were analyzed by removing the red color channel, selecting the area of interest, and measuring the "dark pixels" within the ROI, based on a signal intensity histogram at a wavelength of $0-155 \mathrm{~nm}$. The area of these dark pixels was then expressed as $\mathrm{mm}^{2}$. All procedures conducted on research animals were approved by the Halozyme Institutional Animal Care and Use Committee (IACUC) and complied with the Halozyme IACUC protocol no. 2017-06C. All statistical analyses were carried out using GraphPad Prism version 7.0 (GraphPad Software, San Diego, CA, USA). All 
a
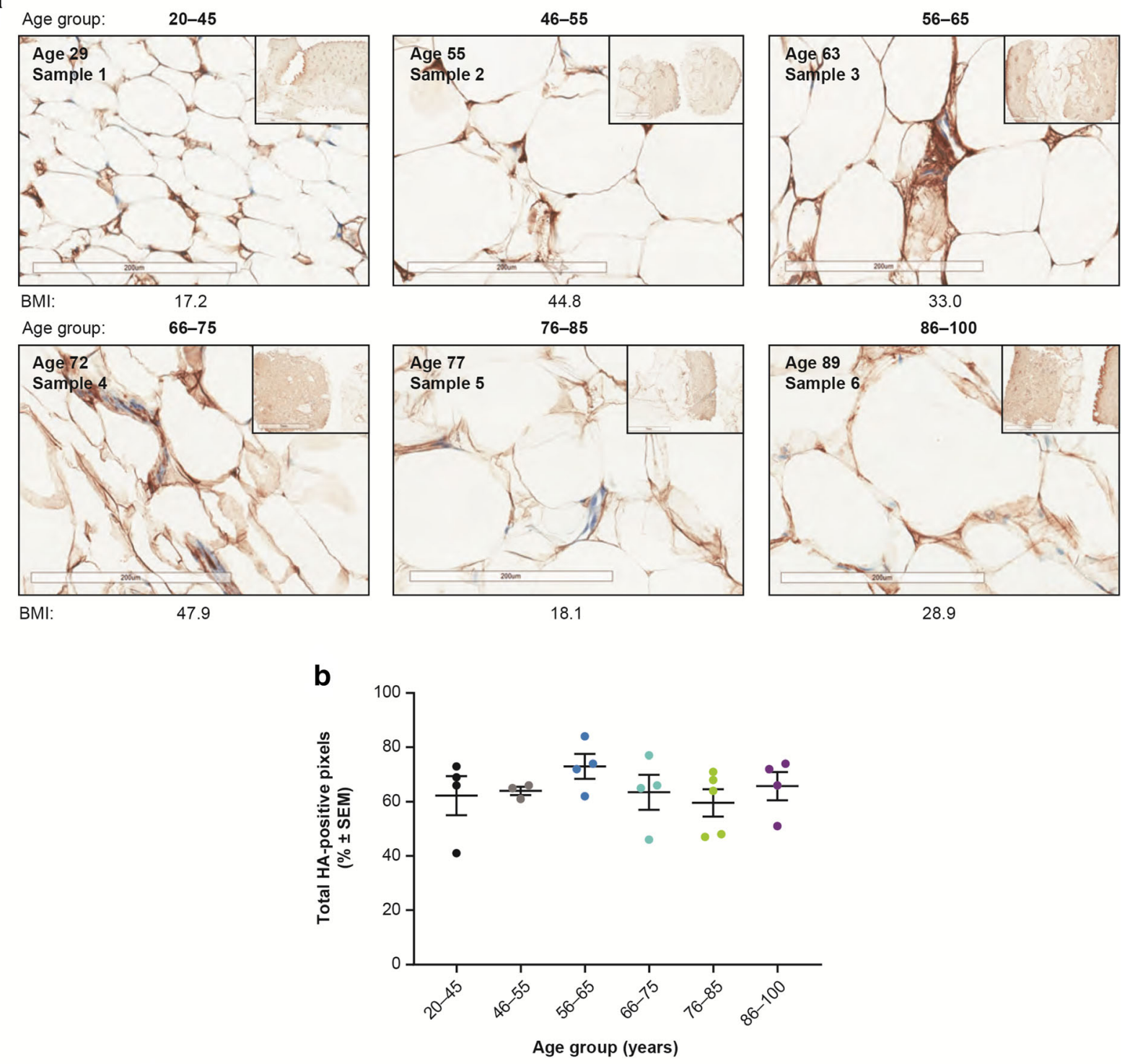

C Age group:

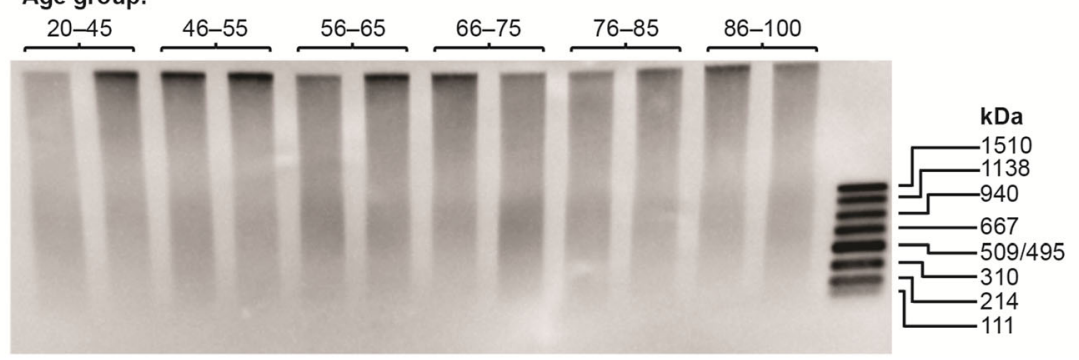


statistical analyses were carried out using GraphPad Prism version 7.0 (GraphPad Software, San Diego, CA, USA).

\section{RESULTS}

\section{HA Distribution and Quantification in Human SC Tissue}

HA distribution and quantification were determined in tissue sections from each age group using a modified TSG-6 probe [27]. All run controls were successful. HA distribution appeared confined to the interstitial space between adipocytes, with similar pericellular presence (Fig. 1a) and similar levels of HA found across all age groups (Fig. 1b). A broad, poly-dispersive profile of HA polymer sizes with similar patterns of distribution was also detected by Western blot analysis of SC HA treated with proteinase K. Substantial levels of high-molecular-weight HA were observed in all age groups (Fig. 1c).

\section{Degradability of HA in Human SC Tissue}

Incubation with rHuPH20 at a clinically relevant concentration $(18 \mu \mathrm{g} / \mathrm{ml} ; 2000 \mathrm{U} / \mathrm{ml})$ resulted in the degradation of all SC HA independent of age, as shown in HA-stained tissue samples from all age groups (Fig. 2a). The extent of HA degradation was shown to be rHuPH20 dependent, as assessed by measuring the percentage of HA-positive pixels in a ROI from the SC space following treatment with $\mathrm{rHuPH} 20$ at a concentration of $18 \mu \mathrm{g} / \mathrm{ml}(2000 \mathrm{U} / \mathrm{ml})$ or $1 \mu \mathrm{g} / \mathrm{ml}(110 \mathrm{U} / \mathrm{ml})$, with more HA removed at the higher concentration (Fig. 2b). To assess HA degradation, HA was quantified after incubating tissue sections with $\mathrm{rHuPH} 20$ at a low concentration $(\sim 11 \mathrm{U} / \mathrm{ml})$ over a 2 -h time course. The degradation profiles were comparable for all age groups (Fig. 2c).

\section{Comparison of Dermal Reconstitution in Young and Elderly Mice}

To assess the effect of aging on dermal HA reconstitution, young $(\sim 1.5$ months $)$ and
Fig. 2 Degradability of subcutaneous HA using rHuPH20 was consistent across age brackets. a Tissue section following incubation with $\mathrm{rHuPH} 20 \quad(18 \mu \mathrm{g} / \mathrm{ml}$; $2000 \mathrm{U} / \mathrm{ml}$ ), stained for HA using a biotinylated TSG6$\Delta$ Hep-Fc probe (HTI-601), followed by hematoxylin counterstain. Any HA present would stain brown. b Quantification of HA levels post-treatment with rHuPH20 showed removal of HA was concentrationdependent. c HA degradation was quantified after incubating tissue sections with $\mathrm{rHuPH} 20(\sim 11 \mathrm{U} / \mathrm{ml})$ over a 2-h time period. $H A$ hyaluronan, $r H u P H 20$ recombinant human hyaluronidase $\mathrm{PH} 20$, SEM standard error mean

elderly ( $>16$ months) mice were treated with an intradermal injection of $\mathrm{rHuPH} 20(0.04 \mathrm{ml}$; $2000 \mathrm{U} / \mathrm{ml})$, and dye dispersion was measured at $2,24,48,72$ and $96 \mathrm{~h}$ post-injection. No differences in dispersion were observed between young and elderly mice across the range of postinjection time points assessed. Both groups of mice showed significant and similar dye dispersion $2 \mathrm{~h}$ post-rHuPH20 treatment when measured at 5 and 20 min post-dye injection compared with baseline (young mice at $5 \mathrm{~min}$ post-dye injection vs. baseline, $p=0.015$; elderly mice at $5 \mathrm{~min}$ post-dye injection vs. baseline, young and elderly mice at $20 \mathrm{~min}$ post-dye injection vs. baseline, $p<0.0001$ ). Furthermore, dye dispersion returned to baseline levels by $24 \mathrm{~h}$ after rHuPH20 treatment for both young and elderly mice, demonstrating reconstitution of the dermis in a similar time frame (Fig. 3).

\section{DISCUSSION}

The SC route of administration is generally preferred by both patients and healthcare providers over IV infusion because of its improved tolerability, less invasive nature, increased convenience for patients and reduced healthcare administration time, costs and resource use $[4,5,7,28-32]$. Owing to the limitations associated with standard SC delivery systems [31, 33], a number of therapeutics currently on the market are subcutaneously co-administered or co-formulated with rHuPH20 [16]. Given that any change to dermal HA levels, distribution, size, degradation or reconstitution time may 

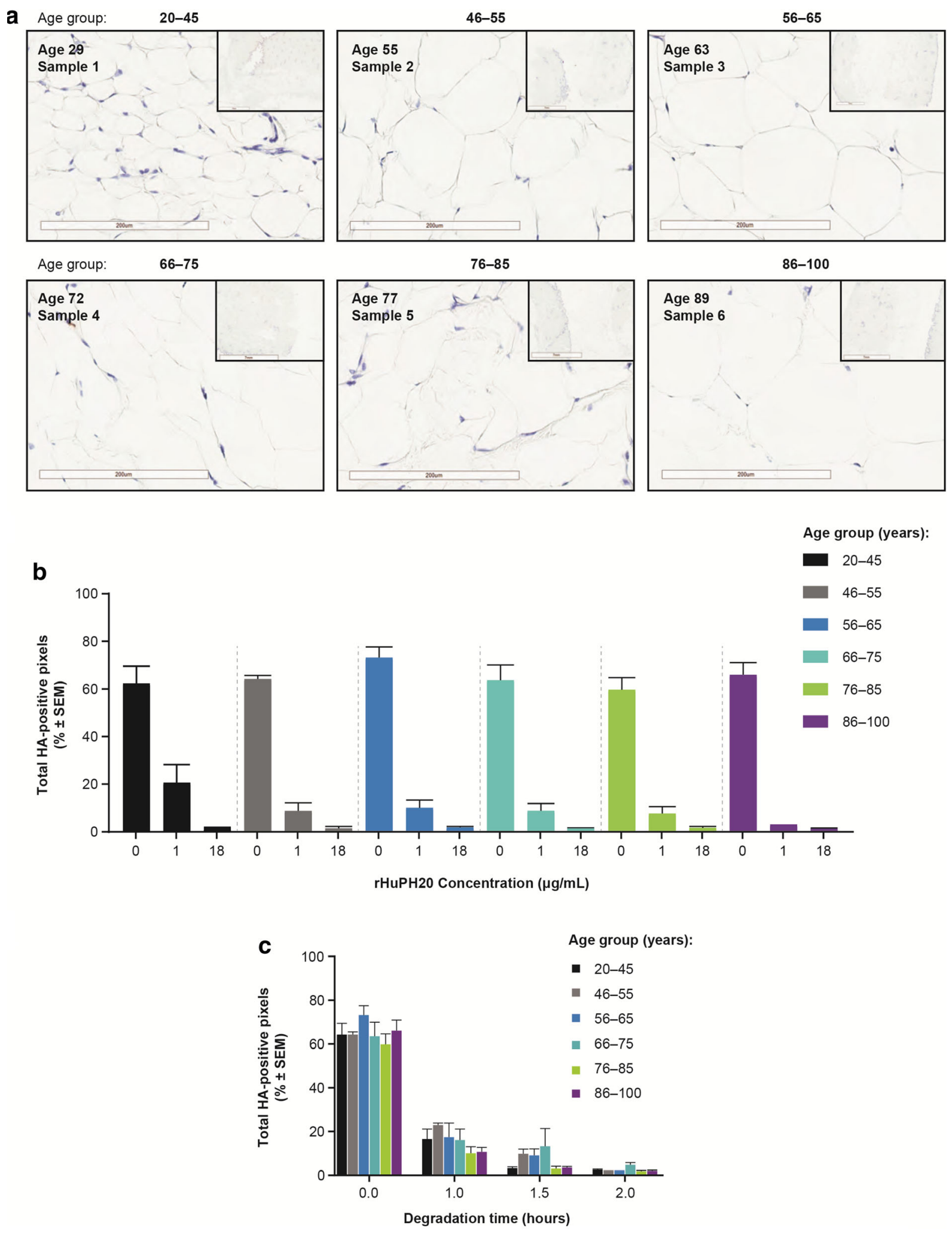

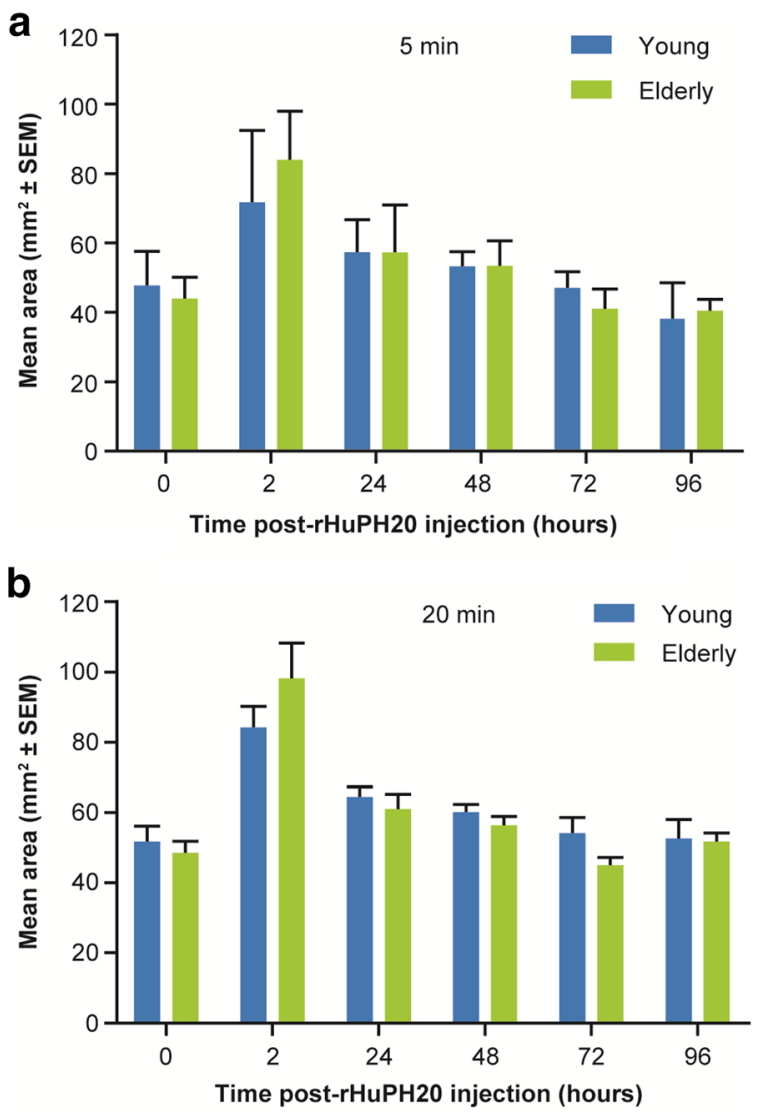

Fig. 3 There were no differences in the time required for dermal reconstitution in mice aged 1.5 months (young) vs. $>16$ months (elderly) using dye dispersion as a pharmacodynamic readout. a Dye dispersion measured at 5 min post-dye administration, at $0,2,24,48,72$ and $96 \mathrm{~h}$ post-rHuPH20 administration. b Dye dispersion measured at $20 \mathrm{~min}$ post-dye administration, at $0,2,24,48,72$ and $96 \mathrm{~h}$ post-rHuPH20 administration. $r H u P H 20$ recombinant human hyaluronidase $\mathrm{PH} 20, S E M$ standard error mean

impact the efficacy of $\mathrm{rHuPH} 20$, this study assessed the effect of aging on these properties.

Our in vitro results in fixed human tissue samples have shown the levels and localization of SC HA to be similar across different age groups. A previous study assessing the polymer size of HA reported that the molecular weight of $\mathrm{HA}$ in the skin is typically in the range of $4000-6000 \mathrm{kDA}$, with lower quantities of some smaller species present [34]; however, there is no consensus in the literature concerning the effect of aging on the size of HA polymers. Meyer and Stern [24] found no significant difference in the sizes of HA polymers across different age groups, while Longas et al. [20] found a decrease in the size of HA polymers with increasing age. Our findings are consistent with those of Meyer and Stern [24], having detected a broad range of HA polymer sizes for all samples and no marked differences in the distribution of HA sizes between age groups. We were unable to provide a clear estimate for the upper range of the HA species present in the samples collected, but the banding clearly showed HA with a molecular weight much higher than the largest of the standards at $1510 \mathrm{kDA}$.

Our results also suggest that any potential effects of aging on the nature of HA did not translate into a loss of rHuPH20 efficacy. This was demonstrated by rHuPH20 concentrationdependent and age-independent degradation of HA from the human SC tissue and there being no observed difference in the time required for dermal reconstitution in young mice aged 1.5 months vs. elderly mice aged $>16$ months. Because of the time constraints associated with growing swine to maturation, a mouse model was used to assess dermal reconstitution of HA. In these murine models, rHuPH20 was administered intradermally, which demonstrates the spreading activity of hyaluronidase and has been used to assess the duration of hyaluronidase activity in humans [12] as well as animals [17]. Dermal reconstitution following rHuPH20 administration was examined in a mouse model using dye dispersion as a pharmacodynamic readout.

This study was potentially limited by the small sample size of available human cadavers and aged athymic mice. In addition, this particular study was the first to use a quantitative histochemistry approach using dye dispersion as an indirect method of assessing HA reconstitution; these results were not confirmed histologically. However, the literature contains examples of similar studies in which reconstitution of HA has been histologically confirmed [35].

\section{CONCLUSIONS}

There is a general preference by patients and HCPs for SC administration, and it is important 
to determine whether age impacts on the ability of rHuPH20 to facilitate SC delivery of co-administered therapeutics. Overall, in human skin samples, no evidence was found for an effect of aging on HA levels, distribution, size or degradation. Moreover, there was no evidence for an effect of aging on dermal reconstitution in young or elderly mice, and there was no apparent loss of rHuPH20 effectiveness with increasing age. Clinical confirmation of these findings could contribute to expanding the patient populations in which rHuPH20 technology can be safely used, but these initial findings suggest that SC delivery of approved therapeutics facilitated by $\mathrm{rHuPH} 20$ should not be impacted by intrinsic aging.

\section{ACKNOWLEDGEMENTS}

The authors thank Sheryl Garrovillo for histology support and all investigators involved in this study.

Funding. The studies were conducted and funded by Halozyme Therapeutics Inc., San Diego, CA, USA, and the data are held by the company. Halozyme Therapeutics Inc. also funded the cost of the journal's rapid service fee. Halozyme follows all current policies established by the International Committee of Medical Journal Editors and Good Publication Practice guidelines (https://annals.org/aim/fullarticle/2424869/ good-publication-practice-communicating-com pany-sponsored-medical-research-gpp3). The sponsor was involved in the study design, collection, analysis, and interpretation of data as well as data checking of information provided in the manuscript. However, ultimate responsibility for opinions, conclusions and data interpretation lies with the authors.

Medical Writing Assistance. Medical writing support, including assisting authors with the development of the outline and initial draft, incorporation of comments and fact checking, was provided by William Stainsby, and editorial support, including figure preparation, formatting, proofreading and submission, was provided by Natalie Morton, both of Paragon, Knutsford, UK, supported by Halozyme Therapeutics Inc.

Authorship. All named authors meet the International Committee of Medical Journal Editors (ICMJE) criteria for authorship for this article, take responsibility for the integrity of the work as a whole and have given their approval for this version to be published.

Prior Presentation. This manuscript is based on work that has been previously presented at the International Society for Hyaluronan Sciences conference, June 9-14, 2019, Cardiff, Wales, UK; Poster number P0080.

Disclosures. David W. Kang is an employee, owns stock and has stock options in Halozyme Therapeutics Inc. Robert J. Connor, Barbara Blouw, Jessica Cowell, Kelly Chen and Chunmei Zhao were formerly employees, owned stock and had stock options in Halozyme Therapeutics Inc. at the time of the study. Jessica Cowell is now an employee of Turning Point Therapeutics.

Compliance with Ethics Guidelines. All procedures conducted on research animals were approved by the Halozyme Institutional Animal Care and Use Committee (IACUC) and complied with the Halozyme IACUC protocol number 2017-06C. We acknowledge the use of human tissues procured by the National Disease Research Interchange (NDRI; protocol number DZEM1) who are supported by NIH grant U42OD111158. All samples were recovered in full respect of the 1964 Declaration of Helsinki and its later amendments, following approval by the University of Pennsylvania Institutional Review Board (IRB) and according to institutional regulations and guidelines regarding informed consent and donor confidentiality.

Data Availability. The datasets during and/ or analyzed during the current study are available from the corresponding author on reasonable request. 
Open Access. This article is licensed under a Creative Commons Attribution-NonCommercial 4.0 International License, which permits any non-commercial use, sharing, adaptation, distribution and reproduction in any medium or format, as long as you give appropriate credit to the original author(s) and the source, provide a link to the Creative Commons licence, and indicate if changes were made. The images or other third party material in this article are included in the article's Creative Commons licence, unless indicated otherwise in a credit line to the material. If material is not included in the article's Creative Commons licence and your intended use is not permitted by statutory regulation or exceeds the permitted use, you will need to obtain permission directly from the copyright holder. To view a copy of this licence, visit http:// creativecommons.org/licenses/by-nc/4.0/.

\section{REFERENCES}

1. Hutin YJ, Hauri AM, Armstrong GL. Use of injections in healthcare settings worldwide, 2000: literature review and regional estimates. BMJ (Clin Res ed). 2003;327:1075.

2. Jin J-F, Zhu L-L, Chen M, et al. The optimal choice of medication administration route regarding intravenous, intramuscular, and subcutaneous injection. Patient Prefer Adherence. 2015;9:923-42.

3. De Cock E, Kritikou P, Sandoval M, et al. Time savings with rituximab subcutaneous injection versus rituximab intravenous infusion: a time and motion study in eight countries. PLOS ONE. 2016;11:e0157957.

4. Wynne C, Harvey V, Schwabe C, et al. Comparison of subcutaneous and intravenous administration of trastuzumab: a phase $\mathrm{I} / \mathrm{Ib}$ trial in healthy male volunteers and patients with HER2-positive breast cancer. J Clin Pharmacol. 2013;53:192-201.

5. Bittner B, Richter W, Schmidt J. Subcutaneous administration of biotherapeutics: an overview of current challenges and opportunities. BioDrugs. 2018;32:425-40.

6. Dychter SS, Gold DA, Haller MF. Subcutaneous drug delivery: a route to increased safety, patient satisfaction, and reduced costs. J Infus Nurs. 2012;35: 154-60.
7. Pivot X, Gligorov J, Muller V, et al. Patients' preferences for subcutaneous trastuzumab versus conventional intravenous infusion for the adjuvant treatment of HER2-positive early breast cancer: final analysis of 488 patients in the international, randomized, two-cohort PrefHer study. Ann Oncol. 2014;25:1979-87.

8. De Cock E, Pan YI, Tao S, et al. Time savings with transtuzumab subcutaneous (SC) injection verse trastuzumab intravenous (IV) infusion: a time and motion study in 3 Russian centers. Value Health. 2014;17:A653.

9. McDonald TA, Zepeda ML, Tomlinson MJ, et al. Subcutaneous administration of biotherapeutics: current experience in animal models. Curr Opin Mol Ther. 2010;12:461-70.

10. Frost GI. Recombinant human hyaluronidase (rHuPH20): an enabling platform for subcutaneous drug and fluid administration. Expert Opin Drug Deliv. 2007;4:427-40.

11. Verzijl N, DeGroot J, Thorpe SR, et al. Effect of collagen turnover on the accumulation of advanced glycation end products. J Biol Chem. 2000;275: 39027-31.

12. Bywaters EGL, Holborow EJ, Keech MK. Reconstitution of the dermal barrier to dye spread after hyaluronidase injection. Br Med J. 1951;2:1178-83.

13. Buhren BA, Schrumpf H, Hoff NP, et al. Hyaluronidase: from clinical applications to molecular and cellular mechanisms. Eur J Med Res. 2016;21:5.

14. DuFort CC, DelGiorno KE, Carlson MA, et al. Interstitial pressure in pancreatic ductal adenocarcinoma is dominated by a gel-fluid phase. Biophys J. 2016;110:2106-19.

15. Kang DW, Jadin L, Nekoroski T, et al. Recombinant human hyaluronidase $\mathrm{PH} 20$ (rHuPH20) facilitates subcutaneous infusions of large volumes of immunoglobulin in a swine model. Drug Deliv Transl Res. 2012;2:254-64.

16. Locke KW, Maneval DC, LaBarre MJ. ENHANZE ${ }^{\circledR}$ drug delivery technology: a novel approach to subcutaneous administration using recombinant human hyaluronidase PH20. Drug Deliv. 2019;26: 98-106.

17. Bookbinder LH, Hofer A, Haller MF, et al. A recombinant human enzyme for enhanced interstitial transport of therapeutics. J Control Release. 2006;114:230-41.

18. Kang DW, Nekoroski TA, Printz MA, et al. Recombinant human hyaluronidase $\mathrm{PH} 20$ (rHuPH20) facilitated subcutaneous delivery of proteins in 
nonclinical models. Control Release Soc Newslett. 2013;30:9-11.

19. Quan T, Fisher GJ. Role of age-associated alterations of the dermal extracellular matrix microenvironment in human skin aging: a mini-review. Gerontology. 2015;61:427-34.

20. Longas MO, Burden JD, Lesniak J, et al. Hyaluronic acid $\mathrm{N}$-deacetylase assay in whole skin. Biomacromol. 2003;4:189-92.

21. Stern R, Maibach HI. Hyaluronan in skin: aspects of aging and its pharmacologic modulation. Clin Dermatol. 2008;26:106-22.

22. Sluke G, Schachtschabel DO, Wever J. Age-related changes in the distribution pattern of glycosaminoglycans synthesized by cultured human diploid fibroblasts (WI-38). Mech Ageing Dev. 1981;16:19-27.

23. Poulsen JH, Cramers MK. Determination of hyaluronic acid, dermatan sulphate, heparan sulphate and chondroitin 4/6 sulphate in human dermis, and a material of reference. Scand J Clin Lab Invest. 1982;42:545-9.

24. Meyer LJ, Stern R. Age-dependent changes of hyaluronan in human skin. J Invest Dermatol. 1994;102:385-9.

25. Tzellos TG, Sinopidis X, Kyrgidis A, et al. Differential hyaluronan homeostasis and expression of proteoglycans in juvenile and adult human skin. J Dermatol Sci. 2011;61:69-72.

26. Jadin L, Pastorino S, Symons R, et al. Hyaluronan expression in primary and secondary brain tumors. Ann Transl Med. 2015;3:80.
27. Jadin L, Huang L, Wei G, et al. Characterization of a novel recombinant hyaluronan binding protein for tissue hyaluronan detection. J Histochem Cytochem. 2014;62:672-83.

28. Awwad S, Angkawinitwong U. Overview of antibody drug delivery. Pharmaceutics. 2018;10:83.

29. Williams EL, Edwards CJ. Patient preferences in choosing anti-TNF therapies-R1. Rheumatology (Oxford). 2006;45:1575-6.

30. Bittner B, Richter WF, Hourcade-Potelleret F, et al. Non-clinical pharmacokinetic/pharmacodynamic and early clinical studies supporting development of a novel subcutaneous formulation for the monoclonal antibody rituximab. Drug Res (Stuttg). 2014;64:569-75.

31. Richter WF, Jacobsen B. Subcutaneous absorption of biotherapeutics: knowns and unknowns. Drug Metab Dispos. 2014;42:1881-9.

32. De Cock E, Pivot $X$, Hauser N, et al. A time and motion study of subcutaneous versus intravenous trastuzumab in patients with HER2-positive early breast cancer. Cancer Med. 2016;5:389-97.

33. Hunter J. Subcutaneous injection technique. Nurs Stand. 2008;22:41-4.

34. Tammi R, Agren UM, Tuhkanen AL, et al. Hyaluronan metabolism in skin. Prog Histochem Cytochem. 1994;29:1-81.

35. Hompesch M, Muchmore DB, Morrow L, et al. Accelerated insulin pharmacokinetics and improved postprandial glycemic control in patients with type 1 diabetes after coadministration of prandial insulins with hyaluronidase. Diabetes Care. 2011;34:666-8. 